\title{
Hand Grip Dynamometry as Prognostic and Predictive Marker in Older Patients With Cancer
}

\section{Ankit Anand* and Ajeet Gajra}

State University of New York, Upstate Medical University, Syracuse, New York, USA

\begin{abstract}
Muscle strength as measured by hand grip dynamometer is a simple, fast and validated test which correlates well with other measures of functional capacity including six-minute walk test, barthel index and independence with activities of daily living. Elderly patients with advanced cancer represent a subset of population that is specially predisposed to loss of muscle strength. Low muscle strength has also been associated with poor quality of life, increased cardiovascular mortality, non-cardiovascular mortality and all-cause mortality, which is independent of diagnosis. This could have important implications for future studies which may use hand grip strength as the sole measure of functional capacity in predicting morbidity and mortality and avoiding complex questionnaires and other time-consuming tests. Jamar hand dynamometer is a commonly used device and is considered an industry standard in current practice. Periodic assessment may help in predicting at risk population and early referral to strength training program may help obviate the negative health effects of low muscle strength. Despite its ease and predictive power however, hand grip dynamometry remains grossly underutilized. Increased awareness of this simple technology will go a long way in making cost effective health related treatment plans for future patients with advanced cancer.
\end{abstract}

\section{Keywords:}

Hand grip dynamometery; Muscle strength; Older patients; Cancer; Outcomes

\section{Introduction}

Muscle strength is a strong determinant in maintaining functional independence in geriatric patients [1]. Sarcopenia, defined as decreased muscle strength, is associated with functional limitations affecting exercise capacity, walking speed and also is a risk factor for falls, frailty, disability and even death $[1,2]$.

Patients with advanced cancer represent a subset that is at significant risk of sarcopenia due to cachexia and skeletal muscle wasting [3]. Irrespective of cancer stage and cachexia, cancer patients have significant impairments in muscle strength as compared to healthy controls when matched by age, sex, body mass index and physical activity level [4]. There are multiple reasons for muscle dysfunction in cancer patients ranging from ageing, cancer pathophysiology, physical inactivity and treatment related toxicity. Cancers are well known to produce pro-inflammatory cytokines such as IFN- $\gamma$, TNF- $\alpha$ and interleukins which can lead to activation of NF- $\kappa \beta$ pathway leading to muscle degradation. Apart from secreting pro-inflammatory cytokines, cancer cells are metabolically active and require a rich supply of glucose, which may starve the muscle cells. Since incidence of cancer increases with age and more than $50 \%$ of all cancers are diagnosed in patients older than 65 years, age related decline in muscle mass can be a confounder for lower muscle strength [4].

Many cancer patients experience significant fatigue which can persist for years after remission. In addition to cancer itself, chemotherapy has significant toxicity ranging from nausea, vomiting, bone marrow suppression, neuropathy and oxidative stress leading to muscle dysfunction. Perceived fatigue in cancer patients is different from physiological fatigue as it is not relieved by rest and apparently worsens with intensification of chemotherapy [5]. All these factors contribute to muscle dysfunction in cancer patients. Studies in patients with locally advanced prostate cancer, early stage colorectal cancer, breast cancer survivors have all revealed lower hand grip strengths compared to age matched healthy controls [4]. The purpose of this review is to increase awareness of this simple to use, cheap and yet useful predictive technique in the clinic. This can go a long way in anticipating toxicity of treatments and saving health care dollars.
Improvement of skeletal muscle function with nutritional support usually precedes an improvement in anthropometric indices and thus tests of skeletal muscle function are more sensitive in determining the effectiveness of treatment in short term [6]. Several powerful tools to measure muscle strength are available, ranging from biolectric impedance, Dual energy X-ray absorptiometry (DEXA) and mid-thigh computed tomography (mCT). DEXA and mCT are very accurate but expensive and complicated tests. Bioelectric impedance on the other hand is a simple test but less reliable, especially in underweight patients [7]

Muscle strength can also be determined by means of isokinetic and hand-held dynamometers. Isokinetic dynamometers can measure muscle strength over multiple planes and velocities and thus are more accurate determinant of actual physical status, however it suffers from lack of portability, cost effectiveness and ease of use [8]. Even though, hand dynamometer measures upper body strength, it functions as a good surrogate for generalized muscle strength as it correlates well with strength of other muscle groups [2].

HGS has been studied in the elderly population and low HGS is associated with a multitude of negative health effects. HGS has been shown in observational studies to be a predictor of all-cause mortality, cardiovascular mortality and non-cardiovascular mortalilty [9]. Assessment for independence in activities of daily living (ADL) is an integral part of any comprehensive geriatric assessment. Several studies have found association between ADLs and HGS. Matsui et al. in their study of 347 patients showed that maximal HGS was associated with independence in ADLs [10]. A large prospective cohort study of over 2400 patients studied HGS over a seven-year period and found HGS as

${ }^{*}$ Corresponding author: Ankit Anand, State University of New York, Upstate Medical University, Syracuse, New York, USA. Tel: 330-860-4216; E-mail: ankitanand55@gmail.com

Received April 30, 2018; Accepted May 08, 2018; Published May 11, 2018

Citation: Anand A, Gajra A (2018) Hand Grip Dynamometry As Prognostic And Predictive Marker In Older Patients With Cancer. J Gerontol Geriatr Res 7: 471. doi:10.4172/2167-7182.1000471

Copyright: $\odot 2018$ Anand A, et al. This is an open-access article distributed under the terms of the Creative Commons Attribution License, which permits unrestricted use, distribution, and reproduction in any medium, provided the original author and source are credited. 
a significant and independent predictor of disability with ADLs [11]. Evidence from observational studies also suggest a link between low HGS and cognitive decline in the elderly. One study reported $43 \%$ decrease in risk of developing Alzheimer's dementia with one unit increase in muscle strength [12]. There seems to be a complex group of interactions between multitude of factors affecting nutrition, muscle strength, vascular disease and mortality [13]. Whether HGS can simply be regarded as a biomarker of ageing needs more research.

HGS has been shown to be a strong predictor of overall quality of life, functional limitations and disability when studied longitudinally [2]. Rantanen et al. in their study of healthy adults, showed that after a 25 year follow up, decreased HGS measured at an average age of 54 years, predicted functional limitations including dependence for activities of daily living, inability to do heavy household work and inability to rise from chair without using arms [2]. Muscle strength thus appears to track over the lifespan of an individual and those with higher baseline muscle strength, have higher reserve before functional incapacity sets in. This effect of muscle strength on late life disability was independent of comorbidities and chronic conditions that patients suffered [2].

Low HGS which functions as a good surrogate of overall muscular strength; is associated with increased mortality and morbidity in diverse samples of population groups which is not affected by social, cultural or economic background $[9,14]$. Specifically, older population and the octogenarians are prone to physical inactivity and low HGS with some reports estimating the prevalence of sarcopenia to be higher than $50 \%$. Reduced muscle strength in turn is associated with physical disability, cognitive decline and mortality $[9,14]$. When divided by tertiles, elderly with the lowest tertile of HGS and those with the highest relative loss of HGS over a span of four-year period had significantly increased mortality as opposed to other groups $[9,14]$. HGS as measured by Hand held dynamometry (HHD) is closely related to ability to walk, serum protein level and ankle brachial index [13]. The fact that HGS is closely related with various measures of functional capacity could have significant implications for future studies which potentially could use HGS as the sole measure of functional capacity.

\section{Literature Review}

\section{Techniques of hand dynamometry}

Hand held dynamometers (HHD) are small, relatively cheap and powerful tools that have emerged as a valid and reliable tool for objectively assessing muscle strength [15]. Jamar hand dynamometer (JHD) is one of the more commonly used tool to assess handgrip strength (HGS) since it is validated, highly reliable, and easy to use [16]. JHD is a hydraulic dynamometer which has excellent inter-instrument reliability with other grip strength measurement devices. JHD is widely cited in medical literature and remains gold standard for comparing other hand-held devices [17]. It is prudent that a standardized approach be taken to take measurements to minimize error and that the same procedure is followed with every patient every time.

JHD has five handle positions; the second position is thought to be most reliable and recommended for routine use. HGS may however be artificially low in this position, especially in women with nails extending one centimeter beyond fingertip [17]. Grip setting at position three is also a standard testing position approved by American Society of Hand Therapists (ASHT) [15]. Each measurement should be taken with the patient seated comfortably in a chair and test arm resting on armrest at 90 degrees with elbow in 90-degree flexion. The other arm should be in a neutral position by the side. Familiarization with the equipment should be offered with one-two trial attempts before measuring HGS
$[15,17]$. Using dominant hand versus non-dominant hand is another topic that merits discussion. One would expect dominant hand to have a higher HGS as opposed to non-dominant hand; however, this varies whether subject is right hand dominant versus left hand dominant. Bohanon in his review of 10 studies found right dominant subjects to be stronger on right but the results were equivocal in left dominant subjects. It may be prudent to avoid comparisons between dominant and non-dominant HGS [18].

Many states use JHD as a standard testing instrument for compensation determinations. JHD is a precision instrument with adjustable handles which can be placed in five grip positions, from 1.375 to 3.375 inches, in half inch increments. The sealed hydraulic system features dual scale readout that displays isometric grip force from 0 to 200 pounds $(90 \mathrm{~kg}$ ) as shown in Figure 1. JHD displays grip force in both pounds and kilograms and automatically retains the highest reading until reset. It is isometric in use and allows almost no perceptible motion of handles, regardless of grip strength resulting in accurate and reproducible results. The average score of the three trials can be compared to the normative data which is provided with the instrument. From a statistical perspective, scores within two standard deviations of the mean are considered within normal limits.

Since patients with very small hands can have difficulty with second position, it may be argued that patients should be instructed to self-adjust HHD such that it fits comfortably relative to hand size [6]. This can however be a deviation from standardized approach. Due to significant variability in HGS with shoulder position, arm position, sitting versus standing, American Society of Hand Therapists (ASHT) has recommended standardized positioning with patients seated comfortably, shoulders adducted and in a neutral position, elbow flexed at $90^{\circ}$, forearm in neutral position and wrist dorsiflexed between 0 and $30^{\circ}$. Standardization could help in comparing inter study results and have wider applicability on general population [17].

Spijkerman et al. studied test retest reliability with four different measurement protocols and found good test retest reliability with all measurement protocols, however significantly different HGS. Highest

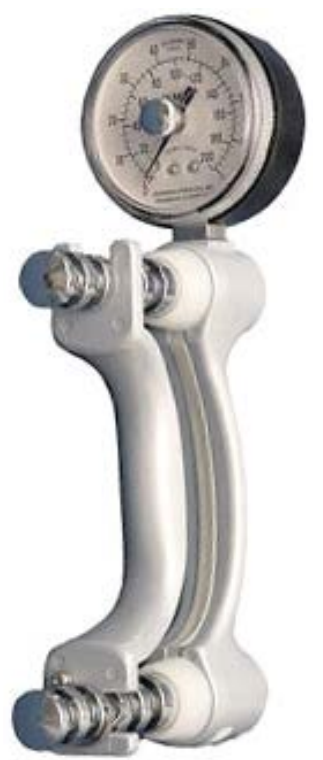

Figure 1: Jamar hand dynamometer. 
HGS was noted when patients were allowed a free comfortable arm position and a challenging stimulus was provided [19].

Current literature supports the use of at least three different trials for measuring HGS; however, it is unclear whether average of three readings or best reading should be used [20]. During repeated HGS measurements however, muscle strength decreases gradually as opposed to measurements after a rest period of 15-60 seconds [17]. Motivation and encouragement have been shown to improve muscle performance acutely, a phenomenon which is widely described [21].

Whatever be the approach, it should be consistent with all patients at all measurements and examiner must ensure that if encouragement is provided, it is consistent across all patients through the study period [20].

\section{Muscle strength and cancer outcomes}

Mortality from cancer has significantly decreased over the past 50 years, however cancer still remains a leading cause of death and cancer survivors experience significant morbidity from complications of cancer treatment and cancer itself [22]. HGS is well known to have positive correlation with overall muscle strength, functional ability and nutritional status in several chronic diseases including advanced cancer. Recently however, HGS has also been shown to be predictive of survival in advanced cancer patients. In a recent study of 203 patients by kilgour et al. patients were divided in cohorts with HGS $<10^{\text {th }}$ percentile and $>50^{\text {th }}$ percentile and outcomes were measured. Study population was heterogeneous and most of the patients were either stage III lung, stage IV lung, pancreatic or gastric cancer, cancers which are more prone to develop cachexia. Cohort with decreased HGS also had significantly lower BMI, poor quality of life and poorer ECOG performance status. Authors concluded that HGS is a potential prognostic determinant of mortality in advanced cancer population. HGS predicted survival independent of gender, age and concurrent cancer treatments [15].

A prospective cohort study by Ruiz et al. studied all-cause mortality, cancer specific mortality in men in relation to muscle strength. Muscle strength was measured separately for upper body and lower body using a standardized strength training protocol with variable resistance weight machines. With an average follow up of 18.9 years, authors concluded that there was significant inverse association between muscle strength and mortality from cancer even after adjusting for age, physical activity, body mass index, co-morbidities, alcohol consumption and cardio-respiratory fitness. Cardio-respiratory fitness along with combination of strength predicts cancer specific mortality. This study was however limited to white men of medium to high socioeconomic status. Also, this study does not clarify what cut off levels will be predictive of increased mortality. Muscle strength does however appear to be a continuum function and moderate level of fitness and strength can have positive outcomes on mortality [23].

Rollins et al. retrospectively studied association between muscle strength and body composition as measured by CT scans in unresectable pancreatic cancer. Median age of group was more than 60 years. Myosteatosis which is defined as attenuated skeletal muscle Hounsfield units was also studied. All patients were evaluated with standard fourth generation multi slice CT helical scanner machine and all images were obtained using same techniques. Measuring body composition with CT analysis may not be the best measure; however it still is comparable with DEXA scan and whole-body MRI. Sarcopenia in this study was not associated with worse survival; interestingly however patients with sarcopenic obesity and myeosteatosis had worse survival. In the cohort with sarcopenia and myosteatosis, median survival was 114 days as opposed to 229 days in cohort without muscle dysfunction [24].
Another study of 62 patients with pancreatic cancer and age $>59$ years noted similar findings. After adjusting in multivariate analysis, hazard ratio of 2.07 was calculated for patients with sarcopenic obesity [25].

Another single center study of 196 patients undergoing resection for hepatic metastasis with colorectal cancer analyzed body composition, muscles strength and compared outcomes with survival. A novel software application from based on MeVisLab version 2.2.1 was used to quantify intra-abdominal fat and skeletal muscle mass using standard diagnostic CT images. Sarcopenia was found in $19.4 \%$ of study population. Five-year disease-free survival for the group with sarcopenia was significantly worse at $15 \%$ versus $28.5 \%$ for the group without sarcopenia, hazard ratio (HR) of 1.88. Central obesity was associated with increased risk of recurrence in men. There was significant selection bias in this study as some patients were removed from study due to lack of CT scans. The cut off values for sarcopenia were again not clear and may vary from one population to another [26].

Villasenor et al. studied sarcopenia in 471 patients with early and locally advanced breast cancer in a prospective observational cohort study, utilizing DEXA scan, which is considered gold standard for measuring sarcopenia. After adjusting for age, race, smoking, alcohol, ethnicity, treatment type, comorbidities, waist circumference, total body fat percentage in a multivariate analysis and a median follow up of 9.2 years, sarcopenia was independently associated increased overall mortality with a hazard ratio of 2.86. Authors also found trend towards increased breast cancer specific mortality, but this was statistically not significant. There were no patients with sarcopenia and obesity in this cohort and hence a conclusion could be made on sarcopenic obesity [27].

Muscle strength as measured by hand grip dynamometer has been shown to be predictive of cancer outcomes in esophageal cancer. Trimodality therapy is considered standard for localized esophageal cancer and esophagectomy itself has considerable morbidity and mortality. Preoperative health status is an important predictor of postoperative recovery. In a cohort of 68 patients with a mean age of 60.7 years who underwent esophagectomy for esophageal cancer, low hand grip strength (defined as $<25 \mathrm{KgF}$ ) was significantly associated with higher perioperative mortality as well as 6-month mortality. Compared to other risk factors like diabetes, low albumin, weight loss; low hand grip strength had the highest relative risk for mortality, with an odds ratio of eight. Hand grip strength less than $25 \mathrm{KgF}$ had a sensitivity of $75 \%$ and specificity of $79 \%$ for predicting mortality. Authors concluded that hand grip strength measurement is cheap, highly predictive and should be used for routine preoperative evaluation of patients undergoing esophagectomy [28].

Another single center study of 101 older patients with stage 3 melanoma who had available CT scans at the time of lymph node dissection were studied for disease free survival and distant disease-free survival as a function of muscle strength. Muscle strength was studied by measuring psoas muscle area and psoas muscle density, which is an objective measure of frailty, but it is not clear how well this correlates with overall muscle strength. On multivariate analysis, low muscle density was significantly associated with decreased distant disease-free survival [29].

Contrary to the above-mentioned studies which found a significant inverse relation of overall survival with muscle strength, a study of 57 patients with metastatic colorectal cancer who underwent treatment with conventional chemotherapy along with bevacizumab did not find an association with overall survival. Muscle strength was measured at baseline and within 3 months of initiating treatment using computed 
tomographic scans at lumbar vertebra L3 level. Authors observed a mean change of weight from $85 \mathrm{~kg}$ to $83 \mathrm{~kg}$ and mean skeletal muscle area dropped from $148 \mathrm{~cm}^{2}$ to $145 \mathrm{~cm}^{2}$ but there was no statistically significant association with adverse overall survival. Patients who gained muscle mass in the intervening period did have a trend towards better survival from 1.51 years to 1.73 years ( $\mathrm{p}$-value=0.33). This study however had selection bias as authors identified 107 patients with metastatic colorectal cancer and 50 patients were removed from analysis due to lack of CT scans. Follow up period was shorter for this study and bevacizumab itself may result in muscle wasting by disrupting PI3K and AKT pathways, which are important skeletal muscle pathways [30].

\section{Muscle strength and other outcome measures}

Whether low HGS is associated with recurrent hospital admissions is a more complex question to answer. While Isaia et al. showed that there was no significant association between HGS and hospital readmissions in a general population over 65 years of age [31], studies in elderly patients with COPD and pneumonia seems to suggest an increased risk of hospital readmission when controlled for other confounders. Further after an episode of hospitalization, HGS significantly decreased in those over 75 years of age $[31,32]$.

Several subjective measures of ADLs correlate with objectively measured HGS. Simard et al. studied 123 elderly patients and developed questionnaire asking 28 questions. Best correlations were seen with, if patients had difficulty opening a jar and how patients compared self-rated grip strength with other people of same age [33]. Matsui et al. studied correlation of HGS with Bartholins index (BI) and found multiple correlations of individual items on BI with HGS, however the correlations were weak, and study included only 347 patients. Authors concluded that study population was small to conclude any inferences, but the study still provided interesting information for future studies [10].

Yilmaz et al. compared exercise capacity, muscle strength and HRQOL measures in early stage versus advanced stage lung cancer patients and found that muscle strength, performance status and HRQOL was significantly lower in patients in advanced lung cancer group as opposed to patients in early stage lung cancer [34].

\section{Interventions}

Arbane et al. conducted a randomized controlled trial comparing twice weekly strength training versus usual care for patients undergoing lung resection for NSCLC and found that exercise training successfully prevented decline in muscle strength as was seen in the control arm. There was however, no change in HRQOL measures and walking distance which returned to baseline at 12 weeks in both the groups. Since long term outcomes were not measured and HRQOL scores returned to baseline in both groups, authors concluded that there was insufficient evidence to recommend strength training for patients undergoing lobectomy over and above, the usual care provided [35]. Cavalheri et al. reviewed three studies involving 178 patients comparing strength training versus usual care and concluded no significant differences seen in muscle strength at the end of study period. Study was however limited by small sample size and methodological limitations and disparities in the studies and authors noted that there was not enough evidence to commend on differences in muscle strength [36].

Advanced stage lung cancer patients who are not candidates for surgery can be treated with chemotherapy, leading to significant improvements in survival. Chemotherapy however, also significantly impacts patient's activity level, consequently muscle strength and quality of life. Henke et al. tested efficacy of a specially designed strength and endurance training on independence and quality of life in advanced lung cancer patients receiving palliative chemotherapy. Only those patients who were able to complete more than $75 \%$ of training sessions were included in statistical analysis and it was noted that there were significant improvements in muscle strength in the intervention group. Interestingly, dropout rate in control group was higher than the intervention group and it was concluded that patients in intervention group had a sense of achievement and enthusiasm with ongoing strength training, leading to low dropout rate from study. It was concluded that strength training resulted in significant improvements in muscle strength, quality of life measures and must be offered to all advanced lung cancer patients receiving palliative chemotherapy [37].

Hwang et al. studied the effects of high-intensity aerobic exercise training although in a different subset of advanced lung cancer patients receiving only targeted therapy. No significant difference on muscle strength and endurance was found in intervention group as opposed to control group. It should however be noted that no strength training was given in this study. Isokinetic test at $180 \mathrm{o}$ which was used to measure muscle strength may not have been sensitive enough to detect the minor changes in muscle strength. Further, both control and treatment groups were small comprising less than 15 patients and almost everyone in control group had requested exercise consultation, likely resulting in non-significant differences in between groups [38].

\section{Discussion}

An alternative approach of neuromuscular electrical stimulation (NMES) of quadriceps in NSCLC patients was studied by Maddocks et al. in a pilot project. Even though trends favored NMES, no significant differences in muscle strength, endurance and activity level were found after 4 weeks of intervention [39].

Aerobic exercise training and resistance training are an integral part of an exercise program. Resistance training specifically decreases all-cause mortality by $33 \%$ and is recommended to be a part of any exercise regimen [40]. Vardar Yagli et al. showed that Yoga along with aerobic exercise regimen is effective in improving muscle strength, functional exercise capacity and fatigue perception. HGS was higher in combined Yoga and exercise group as opposed to aerobic exercise group only [41]. Apart from Yoga, there is evidence from at least three randomized controlled trials about Tai Chi Chuan, a form of aerobic exercise which improves HGS [42].

An ongoing randomized controlled trial from Denmark will provide data on the effectiveness of supervised exercise intervention in patients receiving chemotherapy for advanced lung cancer. Exercise intervention will include strength training with 1.5 -hour sessions twice a week. It will be interesting to see the results of this trial [43].

\section{Conclusion}

It is likely that treatment for advanced cancer and the cancer itself are a cause for significant decline in physical activity, functional status, HRQOL which leads to downward spiral leading to decreased muscle strength and its associated negative health effects. It is important to prevent this downward spiral of quality of life effects. Periodic assessment of muscle strength with HHD should thus be a part of any comprehensive health management plan for geriatric and advanced cancer patients with early referral to a strength training based physical exercise program.

\section{References}

1. Emmelot-Vonk MH, Verhaar HJ, Nakhai Pour HR, Aleman A, Lock TM, et al. (2008) Effect of testosterone supplementation on functional mobility, cognition, and other parameters in older men: a randomized controlled trial. Jama 299: 39-52. 
2. Rantanen T, Guralnik JM, Foley D, Masaki K, Leveille S, et al. (1999) Midlife hand grip strength as a predictor of old age disability. Jama 281: 558-560.

3. Stone CA, Nolan B, Lawlor PG, Kenny RA (2011) Hand-held dynamometry: tester strength is paramount, even in frail populations. J Rehabil Med 43: 808-811.

4. Christensen JF, Jones LW, Andersen JL, Daugaard G, Rorth M, et al. (2014) Muscle dysfunction in cancer patients. Ann Oncol 25: 947-958.

5. Gilliam LA, Clair SDK (2011) Chemotherapy-induced weakness and fatigue in skeletal muscle: the role of oxidative stress. Antioxid Redox Signal 15: 25432563.

6. Schlussel MM, Dos Anjos LA, De Vasconcellos MT, Kac G (2008) Reference values of handgrip dynamometry of healthy adults: a population-based study. Clin Nutr 27: 601-607.

7. Salhi B, Huysse W, Van Maele G, Surmont VF, Derom E, et al. (2014) The effect of radical treatment and rehabilitation on muscle mass and strength: $A$ randomized trial in stages I-III lung cancer patients. Lung Cancer 84: 56-61.

8. Knols RH, Aufdemkampe G, De Bruin ED, Uebelhart D, Aaronson NK (2009) Hand-held dynamometry in patients with haematological malignancies: measurement error in the clinical assessment of knee extension strength. BMC Musculoskelet Disord 10: 31.

9. Leong DP, Teo KK, Rangarajan S, Lopez-Jaramillo P, Avezum A, et al. (2015) Prognostic value of grip strength: findings from the Prospective Urban Rural Epidemiology (PURE) study. Lancet 386: 266-273.

10. Matsui Y, Fujita R, Harada A, Sakurai T, Nemoto T, et al. (2014) Association of grip strength and related indices with independence of activities of daily living in older adults, investigated by a newly-developed grip strength measuring device. Geriatr Gerontol Int 2: 77-86.

11. Snih AS, Markides KS, Ottenbacher KJ, Raji MA (2004) Hand grip strength and incident ADL disability in elderly Mexican Americans over a seven-year period. Aging Clin Exp Res 16: 481-486.

12. Boyle PA, Buchman AS, Wilson RS, Leurgans SE, Bennett DA (2009) Association of muscle strength with the risk of Alzheimer disease and the rate of cognitive decline in community-dwelling older persons. Arch Neurol 66:13391344.

13. Martin-Ponce E, Hernandez-Betancor I, Gonzalez-Reimers E, Hernandez-Luis R, Martinez-Riera A, et al. (2014) Prognostic value of physical function tests: hand grip strength and six-minute walking test in elderly hospitalized patients. Sci Rep 4: 7530.

14. Ling CH, Taekema D, De Craen AJ, Gussekloo J, Westendorp RG, et al. (2010) Handgrip strength and mortality in the oldest old population: the Leiden 85-plus study. Cmaj 182: 429-435.

15. Kilgour RD, Vigano A, Trutschnigg B, Lucar E, Borod M, et al. (2013) Handgrip strength predicts survival and is associated with markers of clinical and functional outcomes in advanced cancer patients. Support Care Cancer 21: 3261-3270.

16. Trutschnigg B, Kilgour RD, Reinglas J, Rosenthall L, Hornby L, et al. (2008) Precision and reliability of strength (Jamar vs. Biodex handgrip) and body composition (dual-energy X-ray absorptiometry vs. bioimpedance analysis) measurements in advanced cancer patients. Appl Physiol Nutr Metab 33: 12321239.

17. Roberts HC, Denison HJ, Martin HJ, Patel HP, Syddall H, et al. (2011) A review of the measurement of grip strength in clinical and epidemiological studies: Towards a standardised approach. Age Ageing 40: 423-429.

18. Bohannon RW (2003) Grip strength: A summary of studies comparing dominant and nondominant limb measurements. Percept Mot Skills 96: 728-730.

19. Spijkerman DC, Snijders CJ, Stijnen T, Lankhorst GJ (1991) Standardization of grip strength measurements. Effects on repeatability and peak force. Scand J Rehabil Med 23: 203-206.

20. Sisto SA, Dyson-Hudson T (2007) Dynamometry testing in spinal cord injury. J Rehabil Res Dev 44: 123-136.

21. Binboga E, Tok S, Catikkas F, Guven S, Dane S (2013) The effects of verbal encouragement and conscientiousness on maximal voluntary contraction of the triceps surae muscle in elite athletes. J Sports Sci 31: 982-988.
22. Schneider CM, Hsieh CC, Sprod LK, Carter SD, Hayward R (2007) Cancer treatment-induced alterations in muscular fitness and quality of life: The role of exercise training. Ann Oncol 18: 1957-1962.

23. Ruiz JR, Sui X, Lobelo F, Morrow JR, Jackson AW, et al. (2008) Association between muscular strength and mortality in men: A prospective cohort study. BMJ 337: a439.

24. Rollins KE, Tewari N, Ackner A, Awwad A, Madhusudan S, et al. (2016) The impact of sarcopenia and myosteatosis on outcomes of unresectable pancreatic cancer or distal cholangiocarcinoma. Clin Nutr 35: 1103-1109.

25. Tan BH, Birdsell LA, Martin L, Baracos VE, Fearon KC (2009) Sarcopenia in an overweight or obese patient is an adverse prognostic factor in pancreatic cancer. Clin Cancer Res 15: 6973-6979.

26. van Vledder MG, Levolger S, Ayez N, Verhoef C, Tran TC, et al. (2012) Body composition and outcome in patients undergoing resection of colorectal liver metastases. Br J Surg 99: 550-557.

27. Villasenor A, Ballard-Barbash R, Baumgartner K, Baumgartner R, Bernstein L, et al. (2012) Prevalence and prognostic effect of sarcopenia in breast cancer survivors: the HEAL Study. J Cancer Surviv 6: 398-406.

28. Chen $\mathrm{CH}, \mathrm{Ho} \mathrm{C}$, Huang $\mathrm{YZ}$, Hung TT (2011) Hand-grip strength is a simple and effective outcome predictor in esophageal cancer following esophagectomy with reconstruction: a prospective study. J Cardiothorac Surg 6: 98.

29. Sabel MS, Lee J, Cai S, Englesbe MJ, Holcombe S, et al. (2011) Sarcopenia as a prognostic factor among patients with stage III melanoma. Ann Surg Oncol 18: 3579-3585.

30. Poterucha T, Burnette B, Jatoi A (2012) A decline in weight and attrition of muscle in colorectal cancer patients receiving chemotherapy with bevacizumab. Med Oncol 29: 1005-1009.

31. Isaia G, Greppi F, Pastorino A, Bersano EM, Rrodhe S, et al. (2013) Predictive effects of muscle strength after hospitalization in old patients. Aging Clin Exp Res 25: 633-636.

32. Martin-Salvador A, Torres-Sanchez I, Saez-Roca G, Lopez-Torres I, Rodriguez-Alzueta E, et al. (2015) Age group analysis of psychological, physical and functional deterioration in patients hospitalized for pneumonia Arch Bronconeumol 51: 496-501.

33. Simard J, Chalifoux M, Fortin V, Archambault MJ, St-Cerny-Gosselin A, et al (2012) Could questions on activities of daily living estimate grip strength of older adults living independently in the community? J Aging Res 2012: 427109

34. Yilmaz E, Ozalevli S, Ersoz H, Yegin A, Onen A, et al. (2013) Comparison of health-related quality of life and exercise capacity according to stages in patients with non-small cell lung cancer. Tuberk Toraks 61: 131-139.

35. Arbane G, Tropman D, Jackson D, Garrod R (2011) Evaluation of an early exercise intervention after thoracotomy for non-small cell lung cancer (NSCLC), effects on quality of life, muscle strength and exercise tolerance: A randomised controlled trial. Lung Cancer 71: 229-234.

36. Cavalheri V, Tahirah F, Nonoyama M, Jenkins S, Hill K (2013) Exercise training undertaken by people within 12 months of lung resection for non-small cell lung cancer. Cochrane Database Syst Rev 7.

37. Henke CC, Cabri J, Fricke L, Pankow W, Kandilakis G, et al. (2014) Strength and endurance training in the treatment of lung cancer patients in stages IIIA/ IIIB/IV. Support Care Cancer 22: 95-101.

38. Hwang CL, Yu CJ, Shih JY, Yang PC, Wu YT (2012) Effects of exercise training on exercise capacity in patients with non-small cell lung cancer receiving targeted therapy. Support Care Cancer 20: 3169-3177.

39. Maddocks M, Lewis M, Chauhan A, Manderson C, Hocknell J, et al. (2009) Randomized controlled pilot study of neuromuscular electrical stimulation of the quadriceps in patients with non-small cell lung cancer. J Pain Symptom Manage 38: 950-956.

40. Dieli-Conwright CM, Orozco BZ (2015) Exercise after breast cancer treatment current perspectives. Breast Cancer: Targets and Therapy 7: 353-362.

41. Vardar-Yagli N, Sener G, Saglam M, Calik-Kutukcu E, Arikan H, et al. (2015) Associations among physical activity, comorbidity, functional capacity, 
Citation: Anand A, Gajra A (2018) Hand Grip Dynamometry As Prognostic And Predictive Marker In Older Patients With Cancer. J Gerontol Geriatr Res 7: 471. doi:10.4172/2167-7182.1000471

Page 6 of 6

peripheral muscle strength and depression in breast cancer survivors. Asian Pac J Cancer Prev 16: 585-589.

42. Pan $\mathrm{Y}$, Yang $\mathrm{K}$, Shi $\mathrm{X}$, Liang $\mathrm{H}$, Zhang F, et al. (2015) Tai chi chuan exercise for patients with breast cancer: a systematic review and meta-analysis. Evid Based Complement Alternat Med 2015: 1-15.
43. Quist M, Langer SW, Rorth M, Christensen KB, Adamsen L (2013) Exhale: exercise as a strategy for rehabilitation in advanced stage lung cancer patients: A randomized clinical trial comparing the effects of 12 weeks supervised exercise intervention versus usual care for advanced stage lung cancer patients. BMC Cancer 13: 477 . 